Research Article

\title{
Some new results on the reciprocal complementary Wiener number of graphs
}

\author{
Ramin Nasiri*, Ali Reza Shojaeifard \\ Department of Mathematics, Faculty of Sciences, Imam Hossein Comprehensive University, Tehran, Iran
}

(Received: 11 December 2021. Received in revised form: 4 January 2022. Accepted: 15 January 2022. Published online: 19 January 2022.)

(C) 2022 the authors. This is an open access article under the CC BY (International 4.0) license (www.creativecommons.org/licenses/by/4.0/).

\begin{abstract}
The reciprocal complementary Wiener number of a graph is one of the applied distance-based topological indices. For a connected graph $G$, this topological index is defined as the sum of the weights $\left(D+1-d_{G}(x, y)\right)^{-1}$ over all unordered pairs of vertices $x$ and $y$ of $G$, where $D$ is the diameter of $G$ and $d_{G}(x, y)$ denotes the distance (that is equal to the length of a shortest path) between $x$ and $y$. In this paper, the reciprocal complementary Wiener number of some cactus graphs is studied.
\end{abstract}

Keywords: reciprocal complementary Wiener number; distance in graphs; cactus chains.

2020 Mathematics Subject Classification: 05C12, 05 C35.

\section{Introduction and preliminaries}

In the mathematical chemistry literature, a molecular descriptor that is calculated from the molecular graph of a chemical compound is known as a topological index. Most of the well-studied topological indices are based on the distances between vertices or/and the degrees of vertices of the molecular graph. The Wiener index is the oldest distance-based topological index and is one of the most-studied topological indices, both from the theoretical point of view as well as from the applications point of view [11]. The first and second Zagreb indices are among the oldest degree-based topological indices $[2,3]$.

Let $G$ be a graph with the vertex set $V(G)=\left\{x_{1}, x_{2}, \ldots, x_{n}\right\}$ and diameter $D$. The reciprocal complementary distance matrix $R C D=\left[r c_{i j}\right]$ of $G$ is an $n \times n$ matrix such that

$$
r c_{i j}=\frac{1}{D+1-d_{G}\left(x_{i}, x_{j}\right)} \quad \text { if } \quad i \neq j,
$$

and 0 otherwise (see [6]). Ivanciuc et al. [4,5] defined the reciprocal complementary Wiener number of the gragh $G$ as:

$$
R C W(G)=\sum_{1 \leq i<j \leq n} r c_{i j}=\sum_{\left\{x_{i}, x_{j}\right\} \subseteq V(G)} \frac{1}{D+1-d_{G}\left(x_{i}, x_{j}\right)} .
$$

If $d(G, k)$ is the number of vertex pairs at distance $k$ in the graph $G$, then we have

$$
R C W(G)=\sum_{k=1}^{D} \frac{d(G, k)}{D+1-k} .
$$

This concept has been successfully applied in the structure-property modeling of the molar heat capacity, standard Gibbs energy of formation and vaporization enthalpy of 134 alkanes $C_{6}-C_{10}$ (see [4]). Recently, we studied the reciprocal complementary Wiener number of various graph operations like join, Cartesian product, composition, strong product, disjunction, symmetric difference, corona product, splice and link of graphs [8]. In [7], we established some new bounds for the reciprocal complementary Wiener number of graphs in terms of the diameter, first and second Zagreb indices, Wiener number, number of vertices, edges and hexagons of graphs. The trees with the smallest, second smallest and third smallest $R C W$, and the unicyclic and bicyclic graphs with the smallest and second smallest $R C W$ were determined in [1]. Zhou et al. [12] gave some properties for the reciprocal complementary Wiener number, especially various upper and lower bounds and a Nordhaus-Gaddum-type result for this invariant. For $4 \leq D \leq n-3$, the unique tree with the minimum reciprocal complementary Wiener number, and the non-caterpillars with the first three smallest values of the reciprocal complementary Wiener number were characterized in [13]. Moreover, Ramane et al. [9] presented some bounds on $R C W$ for line graphs. 
A cactus graph is a connected graph in which any two of its cycles have at most one common vertex. Consequently, each block of this graph is either an edge or a cycle. If all the blocks of a cactus $G$ are cycles of the same size $l$, the cactus is $l$-uniform. A triangular cactus is a 3-uniform cactus, i.e., a cactus in which every block is a triangle. If no triangle of a triangular cactus $G$ has more than two cut vertices, and each cut vertex is shared by exactly two triangle, we say that $G$ is a chain triangular cactus. We denote by $\mathcal{T}_{n}$ the chain triangular cactus graph with $n$ triangles (see Figure 1 ). By replacing the cycles $C_{3}$ in the chain triangular cactus $\mathcal{T}_{n}$ with the cycles $C_{4}$ we obtain cactus graph known as the square cactus chain. The Para-chain square cactus graph, denoted by $\mathcal{Q}_{n}$, is a square cactus chain in which the cut vertices of each of its internal squares are not adjacent (see Figure 2). Also, if the cut vertices of each internal squares of a square cactus chain are adjacent then we call it as the Ortho-chain square cactus graph and denote it by $\mathcal{O}_{n}$ (see Figure 3). Similarly, the chain hexagonal cactus are obtained by replacing the cycles $C_{3}$ with the cycles $C_{6}$ in the chain triangular cactus $\mathcal{T}_{n}$. The Para-chain hexagonal cactus graph, denoted by $\mathcal{L}_{n}$, is a chain hexagonal cactus in which the cut vertices of each of its internal hexagons are at distance three (see Figure 4). Also, if the cut vertices of each of the internal hexagons of a chain hexagonal cactus are at distance two then we call it as the Meta-chain hexagonal cactus graph and denote it by $\mathcal{M}_{n}$ (see Figure 5).

In this paper, we continue our research on the reciprocal complementary Wiener number by determining its values for the above-mentioned cactus chain graphs.

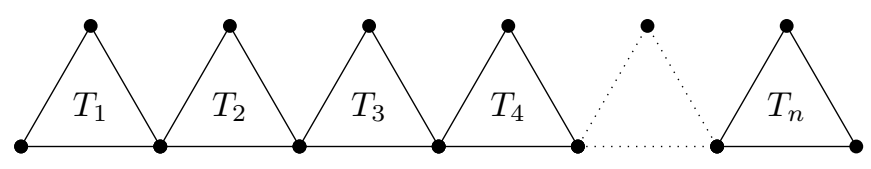

Figure 1: The chain triangular cactus graph $\mathcal{T}_{n}$.
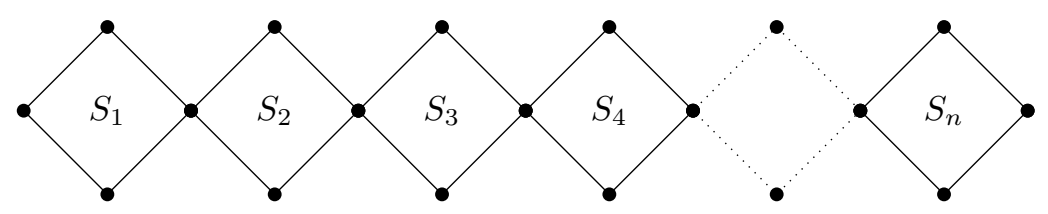

Figure 2: The Para-chain square cactus graph $\mathcal{Q}_{n}$.

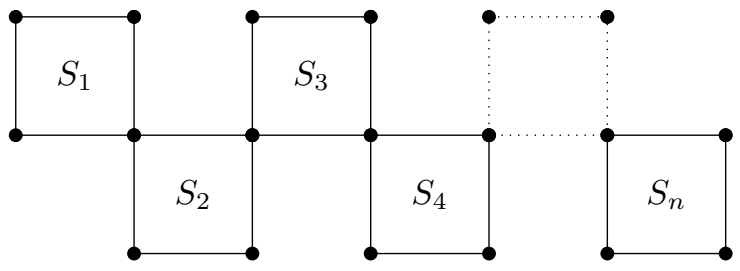

Figure 3: The Ortho-chain square cactus graph $\mathcal{O}_{n}$.

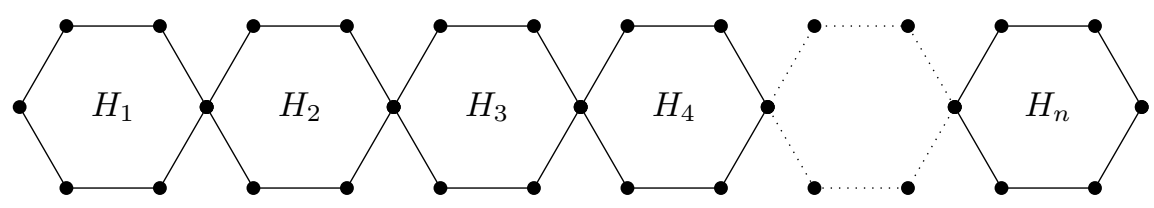

Figure 4: The Para-chain hexagonal cactus graph $\mathcal{L}_{n}$.

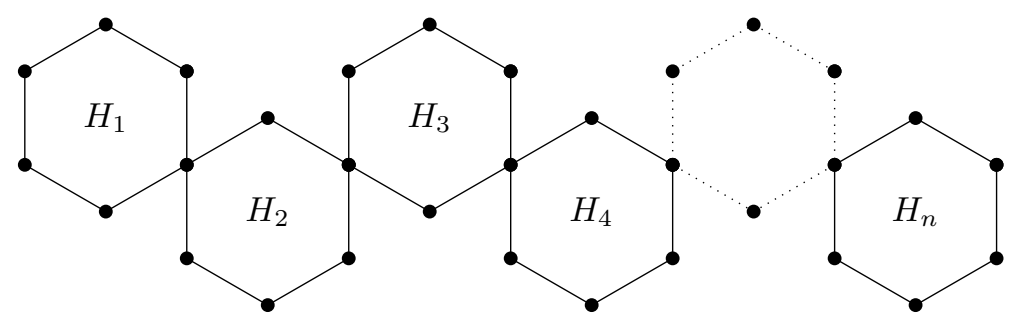

Figure 5: The Meta-chain hexagonal cactus graph $\mathcal{M}_{n}$. 


\section{Main results}

In this section, we study the reciprocal complementary Wiener number of the chain triangular cactus graph, two types of the square cactus chains and two kind of the hexagonal cactus chains.

Theorem 2.1. If $\mathcal{T}_{n}(n \geq 2)$ is the chain triangular cactus graph, then

$$
R C W\left(\mathcal{T}_{n}\right)=4 n-1 .
$$

Proof. By [10], we have

$$
d\left(\mathcal{T}_{n}, k\right)= \begin{cases}3 n & k=1 \\ 4 & k=n \\ 4 n-4 k+4 & 2 \leq k \leq n-1\end{cases}
$$

Therefore,

$$
R C W\left(\mathcal{T}_{n}\right)=\sum_{k=1}^{D=n} \frac{d\left(\mathcal{T}_{n}, k\right)}{n+1-k}=7+\sum_{k=2}^{n-1} \frac{4 n-4 k+4}{n+1-k}=4 n-1 .
$$

Theorem 2.2. Let $\mathcal{Q}_{n}(n \geq 2)$ and $\mathcal{O}_{n}(n \geq 5)$ be the Para-chain and Ortho-chain square cactus graphs, respectively.

(i). For $\mathcal{Q}_{n}$, it holds that

$$
R C W\left(\mathcal{Q}_{n}\right)=\frac{18 n^{2}-13 n}{4 n-2}-\frac{3}{2} \sum_{k=1}^{n-2} \frac{1}{2 k+1} .
$$

(ii). For $\mathcal{O}_{n}$, it holds that

$$
R C W\left(\mathcal{O}_{n}\right)=9 n-9-\frac{30 n^{2}+64 n+24}{n^{3}+3 n^{2}+2 n}-12 \sum_{k=4}^{n-1} \frac{1}{k} .
$$

Proof. By [10], we have

$$
d\left(\mathcal{Q}_{n}, k\right)= \begin{cases}6 n-4 & k=2, \\ 4 & k=2 n-1, \\ 1 & k=2 n, \\ 4 n-2 k+2 & 1 \leq k \leq 2 n-3 \text { and } 2 \nmid k, \\ 5 n-\frac{5}{2} k+1 & 4 \leq k \leq 2 n-2 \text { and } 2 \mid k,\end{cases}
$$

and

$$
d\left(\mathcal{O}_{n}, k\right)= \begin{cases}4 n & k=1, \\ 6 n-4 & k=2, \\ 8 n-12 & k=3, \\ 15 & k=n, \\ 6 & k=n+1, \\ 1 & k=n+2, \\ 9 n-9 k+15 & 4 \leq k \leq n-1 .\end{cases}
$$

By applying the above relations and relation (2), one gets

$$
\begin{aligned}
R C W\left(\mathcal{Q}_{n}\right) & =\sum_{k=1}^{D=2 n} \frac{d\left(\mathcal{Q}_{n}, k\right)}{2 n+1-k} \\
& =3+\frac{6 n-4}{2 n-1}+\sum_{\substack{k=1 \\
2 \nmid k}}^{2 n-3} \frac{4 n-2 k+2}{2 n-k+1}+\sum_{\substack{k=4 \\
2 \mid k}}^{2 n-2} \frac{5 n-\frac{5}{2} k+1}{2 n-k+1} \\
& =2 n+1+\frac{6 n-4}{2 n-1}+\sum_{k=2}^{n-1} \frac{5 n-5 k+1}{2 n-2 k+1}=\frac{18 n^{2}-13 n}{4 n-2}-\frac{3}{2} \sum_{k=1}^{n-2} \frac{1}{2 k+1},
\end{aligned}
$$

as desired. The reciprocal complementary Wiener number of $\mathcal{O}_{n}$ can be computed by a similar way. 
Theorem 2.3. If $\mathcal{Q}_{n}(n \geq 2)$ is the Para-chain graph, then

$$
\frac{34 n^{3}-75 n^{2}+37 n}{(4 n-2)(2 n-3)} \leq R C W\left(\mathcal{Q}_{n}\right) \leq \frac{18 n^{4}-55 n^{3}+77 n^{2}-120 n+48}{2 n(n-2)(2 n-1)},
$$

where each of the equalities holds if and only if $n=3$.

Proof. If $a_{k}$ and $b_{k}$ are real numbers such that $a_{k} \neq 0$, and $m \leq \frac{b_{k}}{a_{k}} \leq M, k=1,2, \ldots l$, then

$$
\sum_{k=1}^{l} b_{k}^{2}+m M \sum_{k=1}^{l} a_{k}^{2} \leq(M+m) \sum_{k=1}^{l} a_{k} b_{k}
$$

with equality if and only if for all $k, 1 \leq k \leq l$, either $b_{k}=m a_{k}$ or $b_{k}=M a_{k}$. This inequality is known as the Diaz-Metcalf inequality. Considering $a_{k}^{-1}=b_{k}=\sqrt{2 k+1}, 1 \leq k \leq n-2$, in this inequality, we have

$$
\sum_{k=1}^{n-2} \frac{1}{2 k+1} \leq \frac{n^{2}-2 n}{3(2 n-3)}
$$

Using this inequality in part $(i)$ of Theorem 2.2, the upper bound for $R C W\left(\mathcal{Q}_{n}\right)$ is obtained. We know from the Cauchy Schwartz inequality that if $a_{k}$ and $b_{k}$ are real numbers, $k=1,2, \ldots l$, then

$$
\left(\sum_{k=1}^{l} a_{k} b_{k}\right)^{2} \leq\left(\sum_{k=1}^{l} a_{k}^{2}\right)\left(\sum_{k=1}^{l} b_{k}^{2}\right)
$$

where the equality holds if and only if $a_{k}=m b_{k}$ for a non-zero constant $m \in \mathbb{R}$. Now, considering $a_{k}^{-1}=b_{k}=\sqrt{2 k+1}$, $1 \leq k \leq n-2$, in the Cauchy Schwartz inequality, we conclude that

$$
\sum_{k=1}^{n-2} \frac{1}{2 k+1} \geq \frac{(n-4)^{2}}{n^{2}-2 n}
$$

Therefore, the lower bound for $R C W\left(\mathcal{Q}_{n}\right)$ is obtained by using this inequality in part (i) of Theorem 2.2. Finally, both equations occur when $k$ is a constant value. This means that $k$ must be 1 and $n$ must be 3 .

Theorem 2.4. If $\mathcal{O}_{n}(n \geq 5)$ is the Ortho-chain square cactus graph, then

$$
\frac{9 n^{5}-3 n^{4}-81 n^{3}+113 n^{2}+514 n+312}{n(n+1)(n+2)(n-1)} \leq R C W\left(\mathcal{O}_{n}\right) \leq \frac{9 n^{5}+21 n^{4}+39 n^{3}+41 n^{2}-78 n-72}{n(n+1)(n+2)(n+3)},
$$

where each of the equalities holds if and only if $n=5$.

Proof. Using similar arguments as in the proof of Theorem 2.3, if we consider $a_{k}^{-1}=b_{k}=\sqrt{k}, 4 \leq k \leq n-1$, in the Diaz-Metcalf and Cauchy Schwartz inequalities then we have

$$
\frac{2(n-4)^{2}}{n^{2}-n-12} \leq \sum_{k=4}^{n-1} \frac{1}{k} \leq \frac{n^{2}-n-12}{8(n-1)} .
$$

Therefore, bounds for $R C W\left(\mathcal{O}_{n}\right)$ is obtained by using this inequalities in part (ii) of Theorem 2.2. Also, both equations occur when $k=4$. This means that $n$ must be 5 .

Theorem 2.5. Let $\mathcal{L}_{n}(n \geq 3)$ and $\mathcal{M}_{n}(n \geq 4)$ be the Para-chain and Meta-chain hexagonal cactus graphs, respectively. Then

$$
\begin{gathered}
R C W\left(\mathcal{L}_{n}\right)=\frac{25}{3} n-2-\frac{4 n-2}{9 n^{2}-9 n+2}-\frac{2}{3} \sum_{k=1}^{n-2} \frac{15 k+8}{9 k^{2}+9 k+2} \text { and } \\
R C W\left(\mathcal{M}_{n}\right)=\frac{25}{2} n+11-\frac{66 n^{3}+55 n^{2}-6 n-4}{4 n^{4}+4 n^{3}-n^{2}-n}-\sum_{k=1}^{n-1} \frac{35 k-8}{4 k^{2}-2 k} .
\end{gathered}
$$


Proof. By [10], we have

$$
d\left(\mathcal{L}_{n}, k\right)= \begin{cases}6 n & k=1, \\ 10 n-4 & k=2, \\ 11 n-8 & k=3, \\ 8 & k=3 n-2, \\ 4 & k=3 n-1, \\ 1 & k=3 n, \\ 8 n-\frac{8 k-8}{3} & 4 \leq k \leq 3 n-5 \text { and } k \equiv 1 \quad(\bmod 3), \\ 8 n-4-\frac{8 k-16}{3} & 5 \leq k \leq 3 n-4 \text { and } k \equiv 2 \quad(\bmod 3), \\ 9 n-3 k+1 & 6 \leq k \leq 3 n-3 \text { and } 3 \mid k,\end{cases}
$$

and

$$
d\left(\mathcal{M}_{n}, k\right)= \begin{cases}6 n & k=1, \\ 10 n-4 & k=2, \\ 11 n-8 & k=3, \\ 12 n-16 & k=4, \\ 16 & k=2 n-1, \\ 10 & k=2 n, \\ 4 & k=2 n+1, \\ 1 & k=2 n+2, \\ 12 n-6 k+10 & 5 \leq k \leq 2 n-3 \text { and } 2 \nmid k, \\ 13 n-\frac{13}{2} k+10 & 6 \leq k \leq 2 n-2 \text { and } 2 \mid k,\end{cases}
$$

By applying the above relations and relation (2), one obtains

$$
\begin{aligned}
R C W\left(\mathcal{L}_{n}\right)= & \sum_{k=1}^{D=3 n} \frac{d\left(\mathcal{L}_{n}, k\right)}{3 n+1-k} \\
= & \frac{23}{3}+\frac{10 n-4}{3 n-1}+\frac{11 n-8}{3 n-2}+\sum_{k \equiv 1}^{3 n-5} \frac{8 n-\frac{8 k-8}{3}}{3 n-k+1} \\
& +\sum_{k=2}^{3 n-4} \frac{8 n-4-\frac{8 k-16}{3}}{3 n-k+1}+\sum_{\substack{k=6 \\
(\bmod 3)}}^{3 n-3} \frac{9 n-3 k+1}{3 n-k+1} \\
= & \frac{8}{3} n+\frac{28}{3}-\frac{4 n-2}{9 n^{2}-9 n+2}+\sum_{k=1}^{n-2}\left(\frac{8 n-8 k-4}{3 n-3 k-1}+\frac{9 n-9 k+1}{3 n-3 k+1}\right) \\
= & \frac{25}{3} n-2-\frac{4 n-2}{9 n^{2}-9 n+2}-\frac{2}{3} \sum_{k=1}^{n-2} \frac{15 k+8}{9 k^{2}+9 k+2},
\end{aligned}
$$

as desired. The reciprocal complementary Wiener number of $\mathcal{M}_{n}$ can be computed by a similar way.

\section{Acknowledgement}

The authors are grateful to the anonymous referees for their useful and constructive suggestions.

\section{References}

[1] X. Cai, B. Zhou, Reciprocal complementary Wiener numbers of trees, unicyclic and bicyclic graphs, Discrete Appl. Math. 157 (2009) 3046-3054.

[2] I. Gutman, B. Ruščić, N. Trinajstić, C. F. Wilcox, Graph theory and molecular orbitals. XII. Acyclic polyenes, J. Chem. Phys. 62 (1975) 3399-3405.

[3] I. Gutman, N. Trinajstić, Graph theory and molecular orbitals. Total $\varphi$-electron energy of alternant hydrocarbons, Chem. Phys. Lett. 17 (1972) $535-538$. 
[4] O. Ivanciuc, QSAR comparative study of Wiener descriptors for weighted molecular graphs, J. Chem. Inf. Comput. Sci. 40 (2000) 1412-1422.

[5] O. Ivanciuc, T. Ivanciuc, A. T. Balaban, The complementary distance matrix: a new molecular graph metric, ACH Models Chem. 137 (2000) 57-82.

[6] D. Janežič, A. Miličevič, S. Nikolič, N. Trinajstič, Graph Theoretical Matrices in Chemistry, Univ. Kragujevac, Kragujevac, 2007.

[7] R. Nasiri, A. Nakhaei, A. R. Shojaeifard, The reciprocal complementary Wiener number of graphs, Tamkang J. Math. 50 (2019) 371-381.

[8] R. Nasiri, A. Nakhaei, A. R. Shojaeifard, The reciprocal complementary Wiener number of graph operations, Kragujevac J. Math. 45 (2021) 139-154.

[9] H. S. Ramane, V. V. Manjalapur, Reciprocal Wiener index and reciprocal complementary Wiener index of line graphs, Indian J. Discrete Math. 1 (2015) 23-32.

[10] A. Sadeghieh, S. Alikhani, N. Ghanbari, A. J. M. Khalaf, Hosoya polynomial of some cactus chains, Cogent Math. 4 (2017) \#1305638.

[11] H. Wiener, Structural determination of paraffin boiling points, J. Amer. Chem. Soc. 69 (1947) 17-20.

[12] B. Zhou, X. Cai, N. Trinajstić, On reciprocal complementary Wiener number, Discrete Appl. Math. 157 (2009) 1628-1633.

[13] Y. Zhu, F. Wei, F. Li, Reciprocal complementary Wiener numbers of non-caterpillars, Appl. Math. 7 (2016) 219-226. 